\title{
Decreasing the leakage of continuous femoral nerve catheter fixation using 2 - octyl cyanoacrylate glue (Demabond®): A Randomized Controlled Trial study
}

Theerawat Chalacheewa ( $\sim$ drtheerawat@gmail.com )

Vanlapa Arnuntasupakul

Mahidol University Faculty of Medicine Ramathibodi Hospital

\section{Lisa Sangkum}

Mahidol University Faculty of Medicine Ramathibodi Hospital https://orcid.org/0000-0002-4638-0198

\section{Rungrawan Buachai}

Mahidol University Faculty of Medicine Ramathibodi Hospital

jiravud Chanvitayapongs

: Mahidol University Faculty of Medicine Ramathibodi Hospital

\section{Research article}

Keywords: Continuous peripheral nerve catheters, pericatheter leakage, catheter displacement,

Dermabond

Posted Date: October 13th, 2020

DOl: https://doi.org/10.21203/rs.3.rs-41791/v3

License: (c) (i) This work is licensed under a Creative Commons Attribution 4.0 International License.

Read Full License 


\section{Abstract}

Background: Continuous peripheral nerve catheters (CPNCs) have been used for postoperative pain relief. A common problem encountered with CPNCs is pericatheter leakage which can lead to dressing adhesive failure. Frequent dressing changes increase the risk of catheter dislodgement and infections. Adhesive glue is effective in securing peripheral nerve catheter and decreasing leakage around the catheter insertion site. This study is aimed to evaluate the incidence of pericatheter leakage by using fixation with 2-octyl cyanoacrylate glue (Demabond ${ }^{\circledR}$ ) compared to sterile strip.

Methods: Thirty patients undergoing unilateral total knee arthroplasty (TKA) with continuous femoral nerve catheter for postoperative analgesia were randomized to catheter fixation with 2-octyl cyanoacrylate glue (Demabond ${ }^{\circledR}$ ) group or sterile strip group. The primary outcome was the incidence of pericatheter leakage. Secondary outcomes included: frequent of catheter displacement, difficulty of catheter removal, pain score and patient satisfaction.

Results: The incidence of pericatheter leakage at 24 and 48 hours was $0 \%$ versus $93 \%$ and $0 \%$ versus $100 \%$ in the Demabond and sterile strip group, respectively $(P<0.001)$. The incidence of displacement at 24 and 48 hours was $6.7 \%$ versus $93.3 \%$ and $6.7 \%$ versus $100 \%$, respectively $(P<0.001)$. There was no difference in numeric rating scale, difficulty of catheter removal as well as satisfaction scores between groups.

Conclusion: Catheter fixation with 2-octyl cyanoacrylate glue (Demabond ${ }^{\circledR}$ ) decreased the incidence of pericatheter leakage as well as catheter displacement over 48 hours when compared to sterile strip fixation.

\section{Background}

Continuous peripheral nerve catheters (CPNCs) provide sustained postoperative pain relief with opioid sparing effect, improved rehabilitation and patient satisfaction (1-3). The challenge to maintain CPNCs function is securing the catheter in the correct position, especially in the freely mobile sites of the neck and limb. Moreover, by preventing pericatheter leakage, the risk of dislodgement and infection deceases (4). Several methods to secure catheters have been studied, such as suturing, cutaneous suture (5) and retrograde subcutaneous tunneling (6). However, accidental dislodgment and pericatheter leakage are still a frequent problem.

In our institute, patients undergoing TKA received continuous femoral nerve blocks with stimulating catheters for postoperative analgesia. We used stimulating catheter allow for confirmation of catheter tip location near the target nerve. Since stimulating catheter are placed using catheter through needle method, this causes the needle puncture hole diameter to become larger than the catheter, therefore pericatheter leakage is a significant problem in our patients. Previous studies (7-9) showed that adhesive glue was an effective method for peripheral nerve catheter fixation; however, leakage around the 
pericatheter was only their secondary outcome. Fixation of the peripheral catheter with 2ocylcyanoacrylate (Dermabond $\AA$ ) may improve leakage by sealing effect at the puncture site and securing the continuous femoral nerve catheter with 2-ocylcyanoacrylate (Dermabond $\AA$ ) would reduce the incidence of pericatheter leakage within the first 48 hours. The primary aim of this study is to evaluate the incidence of pericatheter leakage by using sterile stripe or 2-ocylcyanoacrylate (Dermabond $\circledast$ ) in continuous femoral catheter. The secondary outcomes were catheter displacement, numeric rating scale score (NRS), difficulty of catheter removal, and patient satisfaction with analgesia.

\section{Methods}

\section{Study participants}

After receiving approval by the Ramathibodi hospital research ethics board. This trial was registered on Thai clinical trial registry: TCTR20200228002, registered 24 February 2020-Retrospectively registered, http://www.clinicaltrials.in.th/index.php?

$\mathrm{tp}=$ regtrials\&menu=trialsearch\&smenu=Isresult\&task=search\&task2=Is. Reporting of this study was done by adhering to the Consolidated Standards of Reporting Trials (CONSORT) statement for reporting of randomized trial. Written informed consent was obtained from all participants. We enrolled 30 adult patients aged 40-80 years, with American Society of Anesthesiologists physical status I-IV who were scheduled to undergo total knee arthroplasty (TKA). We excluded patients who refused to participate in the study, patients with contraindications for to performing femoral nerve blocks and spinal anesthesia (e.g. localized infection at puncture site, history of the femoral nerve neuropathy, history of local anesthetic drug/ adhesive glue allergy)

\section{Randomization}

Our research coordinator, who was not involve in the study, performed computer-generated simple 1:1 ratio to 2-octyl cyanoacrylate glue (Demabond $\AA$ ) (Dermabond group) or normal practice fixation (Control group). Allocation of patients to each group was concealed in a sealed envelope. The anesthesiologist who performed femoral nerve block opened the envelope just before the time of block performance.

\section{Femoral nerve block performance}

For patients undergoing unilateral TKA, femoral sheath catheters were inserted pre-operatively using a nerve stimulator., A Stimulong ${ }^{\circledR} 18$-gauge needle was insertedat $1.5 \mathrm{~cm}$ lateral to the femoral artery. The nerve stimulator was initially set at $2 \mathrm{~Hz}$ and $1.5 \mathrm{~mA}$. When quadriceps contraction was detected, the current was decreased, and the needle position was optimized for contraction at a current output of 0.2$0.5 \mathrm{~mA}$. The Stimulong ${ }^{\circledR} 20$-gauge catheter was attached to the nerve stimulator and the catheter slowly threaded until a depth of $3-5 \mathrm{~cm}$ from the needle tip while maintaining quadriceps contraction at a current $\leq 0.5 \mathrm{~mA}$. The needle was withdrawn, and the patients were divided into two groups: Dermabond group and Control group. In the Demabond group, catheter is sealed with 2-octyl cyanoacrylate liquid adhesive (Dermabond ${ }^{\circledR}$ ), and the area is covered with transparent dressing (Tegaderm ${ }^{\mathrm{TM}}$ ). In the control 
group, the catheter is secured with sterile strip, and the area is covered with transparent dressing (Tegaderm $^{\mathrm{TM}}$ ) (figure 1).

Intra-operative, a spinal anesthesia was performed with a 27-gauge quincke needle using isobaric bupivacaine $10-15 \mathrm{mg}$ at the second to the fourth lumbar levels. No local anesthetics were given through the femoral catheter during surgery. In the recovery room, the femoral catheter was bolus with $15 \mathrm{ml}$ of bupivacaine $0.125 \%$ and then began a continuous infusion with bupivacaine $0.08 \%$ at $5 \mathrm{~mL} / \mathrm{h}$. Every patient received acetaminophen $1000 \mathrm{mg}$ (every 6 hours) and naproxen $250 \mathrm{mg}$ (every 8 hours) for 3 days. If postoperative pain score was greater than four out of 10 , IV morphine $3 \mathrm{mg}$ were administered as rescue analgesia.

\section{Postoperative follow-up}

The incidence of pericatheter leakage, catheter displacement, difficult catheter removal, pain score and patient satisfaction were assessed by the acute pain service nurse.Pericatheter leakage was classified into 4 grades: 1) no leakage, 2) mild: fluid around puncture site but no fluid collection under transparent dressing $\left(\right.$ Tegaderm $\left.^{\mathrm{TM}}\right)$, 3) moderate: fluid collection seen under transparent dressing (Tegaderm ${ }^{\mathrm{TM}}$ ) but did not leak outside , 4) Severe; fluid leak outside of the transparent dressing (Tegaderm ${ }^{\mathrm{TM}}$ ).

The catheter displacement was defined as catheter migration from initial recorded depth of insertion. Catheter dislodgement was defined as complete catheter removal from the skin. Catheter removal was reported as "easy" or "difficult". Post-operative pain was assessed in the postanesthetic care unit (PACU), then 24 hours and 48 hours; using a 10-point numerical rating scale. Patient satisfaction with analgesia using $0-10$ scale with $0=$ vary dissatisfied and $10=$ completely satisfied (excellent).

\section{Statistical Methodology}

Demographic characteristics of the subjects in each randomized controlled study was analyzed. Continuous variables are reported using mean and standard deviation values or median and range values. Categorical variables are presented using counts and percentages and tested using Chi-Square or Fisher exact test, as appropriate. Continuous variables were tested for normality with the Shapiro-Wilk test. Student T-test or Mann-Whitney test was used for group comparisons as appropriate. P-value $<0.05$ implied statistical significance. The statistical software SPSS 20.0 for Windows was used for data analyses.

The sample size was calculated assuming a catheter leakage rate of $50 \%$ with standard fixation techniques based on previous literature (9) and institutional pilot data to have at least $80 \%$ power to see a clinically relevant reduction to zero percentage with a 2-sided type I error rate of 0.05 . A sample size of 11 subjects in each arm of each group was considered adequate. Additional 4 subjects per group was recruited to prevent loss of power because of early withdrawal or protocol violations. Thus, 15 subjects per group was the derived sample size. 


\section{Results}

The distribution and allocation of the patients are outlined in the CONSORT flow diagram (figure 2).

In both groups, there was no significant difference in the sex, age, body mass index (BMI), and ASA physical status of the subjects (Table 1 ).

The incidence of pericatheter leakage at 24 hour and 48 hours was significant lower in Dermabond group compared to control group (At 24 hours: $0 \%$ versus $93.33 \%, p<0.001,48$ hours: $0 \%$ versus $100 \%, p<$ 0.001). The incidence of catheter displacement was significantly lower in Dermabond group compared to control group (At 24 hours: $6.7 \%$ versus $73.3 \%, p<0.001$, 48 hours: $6.7 \%$ versus $93.3 \%, p<0.001$ ) as well as incidence of CPNCs dressing changes. There was no difference numeric rating scale at 24 and 48 hours between groups (24 hours NRS: 2 (0-7) versus 2 (0-8), P= 0.54), 48-hours NRS: 2 (0-7) versus 2 (0$7), P=0.81$ ) as well as 24 hours morphine consumption (Morphine consumption: Dermabond vs. control group: 3 (0-5) vs. $2(0-6), P=0.512$ ) and satisfaction score (Satisfaction score: Dermabond vs. control group: $9.13 \pm 1.13$ vs $9.07 \pm 1.28, P=0.881$ ) (Table 2 ). In both groups, there was no incidence of dislodgement as well as difficulty of catheter removal.

\section{Discussion}

This prospective randomized trial study reveals a significant improvement of pericatheter leakage by using 2-octylcyanoacrylate (Dermabond $\AA$ ) fixation when compared with sterile strips. It also decreased the occurrence of catheter displacement and frequent dressing changes while maintaining high quality pain control and satisfaction scores.

2-octylcyanoacrylate (Dermabond $\AA$ ) is a liquid monomer that spontaneously polymerizes in the presence of moisture to create a waterproof bond with the surrounding epidermis. Several studies have compared this surgical adhesive against sutures for the closure of lacerations in both trauma and elective surgery. Therefore, many studies have been evaluated efficacy of dermabond on CPNCs. Our data clearly reveals that 2-octylcyanoacrylate (Dermabond $\AA$ ) improve pericatheter leakage by sealing effect at the puncture site and secures the catheter tightly by the skin.

Gurnaney et al. reported the incidence of pericatheter leakage before and after using Dermabond as a catheter fixation protocol in CPNCs. The incidence of catheter leakage deceased from $3.87 \%$ to $0.56 \%$ (10). David B et al. conducted a study comparing continuous interscalene catheter fixation between Dermabond and Mastisol, the incidence of pericatheter leakage was only their secondary outcome. They found that Dermabond group had incidence of peri-catheter leakage at 24 and 48 hours 3\% (1/31) and $0 \%(0 / 27)$, P-value $<0.001$. Moreover, patient in dermabond group had lower incidence of catheter migration than Mastisol group (91\% vs. 65\%, P-value 0.012).

Regarding a higher leakage rate in control group when compared to oyher studies, we attribute this to the catheter through needle technique during placement and the groin area being a highly mobile region. This 
prospective randomized trial systematically followed perineural catheter leakage in continuous femoral nerve block over 48 hours after TKA. The results reveal Dermabond was more effective in preventing perineural catheter leakage than using sterile strips. Moreover, the use of Dermabond reduced pericatheter bleeding from the puncture site. There was no difficulty in catheter removal, suggesting that despite a waterproof bond with the epidermis, Dermabond does not increase the difficulty of catheter removal at the time of planned discontinuation. Even though the cost of Dermabond is greater sterile strips, the indirect costs of failed CPNBs from additional nursing care and medications may be even higher. In this study, we found no leakage in the Dermabond group; thus, there was no need to change the dressing and meant less nursing care. From the result, fixation CPNCs with Dermabond maybe useful in ambulatory setting. Since the incidence of pericatheter leakage, displacement and dislodgment is reduced and will require less patient self-care or dis-comfort at home.

Our study has limitations. First, in our study the assessor and patient were not blinded to the fixation technique, potentially contributing to observer bias in the study. However, our leakage and displacement criteria are objectively evaluated. Thereby, reducing the potential for observer bias. Second, there are several perineural catheter systems available in the market, and these results may not be generalized to all types of peripheral nerve block catheters, such as catheter over needle technique. Since our institute mainly place CPNCs using the catheter-through-needle technique, we found fixation using dermabond significantly reduces the occurrence of pericatheter leakage. Third, the results of our study were obtained from a single center, which has vast experience in catheter care process, this experience must be considered when attempting in different clinical setting. Lastly, our study did not include the incidence of leakage or displacement after 48 hours; as our post-operative knee replacement protocol stopped local anesthetic infusion on postoperative day 2 to enhance patient muscle strength for ambulation.

\section{Conclusion}

Fixation catheter with 2-octylcyanoacrylate (Dermabond $\AA$ ) reduced the incidence of pericatheter leakage as well as catheter displacement over 48 hours in a continuous femoral block when compared to sterile strip.

\section{Abbreviations}

BMI: Body mass index

CPNCs: Continuous peripheral nerve catheters (CPNCs)

NRS: Numeric rating scale score

PACU: Post anesthetic care unit

TKA: Total knee arthroplasty 


\section{Declarations}

\section{Ethics approval and consent to participate}

Written informed consent was obtained from all participants. The study was approved by the Ramathibodi hospital research ethics board. This trial was registered on Thai clinical trial registry: TCTR20200228002, registered 24 February 2020- Retrospectively registered, http://www.clinicaltrials.in.th/index.php? $t p=$ regtrials\&menu=trialsearch\&smenu=lsresult\&task=search\&task $2=\mid s$.

Consent for publication: Not applicable

\section{Availability of data and materials:}

The datasets during and/or analyzed during the current study available from the corresponding author on reasonable request.

Competing interests: The authors declare that they have no competing interests

Funding: Not applicable

\section{Authors' contributions}

Conceptualization: T.C., V.A.; Methodology: L.S., T.C.; Formal analysis and investigation: T.C., L.S., R.B.; Writing - original draft preparation: T.W., V.A., L.S.; Writing - review and editing: T.W., J.C

Acknowledgements: Not applicable

\section{References}

1. Ilfeld BM. Continuous peripheral nerve blocks: a review of the published evidence. Anesth Analg. 2011;113(4):904-25.

2. Capdevila $X$, Ponrouch $M$, Choquet 0 . Continuous peripheral nerve blocks in clinical practice. Curr Opin Anaesthesiol. 2008;21(5):619-23.

3. Chelly JE, Ghisi D, Fanelli A. Continuous peripheral nerve blocks in acute pain management. $\mathrm{Br} J$ Anaesth. 2010;105 Suppl 1:i86-i96.

4. Capdevila X, Bringuier S, Borgeat A. Infectious risk of continuous peripheral nerve blocks. Anesthesiology. 2009;110(1):182-8.

5. Klein SM, Greengrass RA, Gleason DH, Nunley JA, Steele SM. Major ambulatory surgery with continuous regional anesthesia and a disposable infusion pump. Anesthesiology. 1999;91(2):563-5.

6. Borgeat A, Tewes E, Biasca N, Gerber C. Patient-controlled interscalene analgesia with ropivacaine after major shoulder surgery: PCIA vs PCA. Br J Anaesth. 1998;81(4):603-5. 
7. Auyong DB, Cantor DA, Green C, Hanson NA. The Effect of Fixation Technique on Continuous Interscalene Nerve Block Catheter Success: A Randomized, Double-Blind Trial. Anesth Analg. 2017;124(3):959-65.

8. Klein SM, Nielsen KC, Buckenmaier CC 3rd, Kamal AS, Rubin Y, Steele SM. 2-octyl cyanoacrylate glue for the fixation of continuous peripheral nerve catheters. Anesthesiology. 2003;98(2):590-1.

9. Switzer EF, Dinsmore RC, North JH Jr. Subcuticular closure versus Dermabond: a prospective randomized trial. Am Surg. 2003;69(5):434-6.

10. Gurnaney H, Kraemer FW, Ganesh A. Dermabond decreases pericatheter local anesthetic leakage after continuous perineural infusions. Anesth Analg. 2011;113(1):206.

\section{Tables}

Table 1 Patient's demographic data

\begin{tabular}{|lllc|}
\hline & Dermabond group (15) & $\begin{array}{l}\text { Control group } \\
(15)\end{array}$ & P-value \\
\hline Male/Female, $\mathrm{n}$ & $0 / 15$ & $3 / 12$ & 0.224 \\
\hline Age $(\mathrm{y})$, mean \pm SD & $68.2 \pm 7.5$ & $66.33 \pm 6.77$ & 0.48 \\
\hline $\mathrm{BMI}\left(\mathrm{kg} \cdot \mathrm{m}^{-2}\right)$, mean \pm SD & $25.99 \pm 3.67$ & $27.41 \pm 3.26$ & 0.272 \\
\hline ASA I/II/III, $\mathrm{n}$ & $0 / 8 / 7$ & $1 / 8 / 6$ & $>0.999$ \\
\hline
\end{tabular}

ASA: American society of anesthesiologist, BMI: Body mass index, SD: Standard deviation

Table 2 Catheter and subject related outcomes for Dermabond compared with control group 


\begin{tabular}{|c|c|c|c|}
\hline & Dermabond group & Control group & P-value \\
\hline \multicolumn{4}{|l|}{ Leakage, n (\%) } \\
\hline 24 hours after operation & $0(0 \%)$ & $14(93.33 \%)$ & $<0.001 *$ \\
\hline Mild & - & $2(13.33 \%)$ & \\
\hline Moderate & - & $2(13.33 \%)$ & \\
\hline Severe & - & $10(66.67 \%)$ & \\
\hline 48 hours after operation & $0(0 \%)$ & $15(100 \%)$ & $<0.001 *$ \\
\hline Mild & - & $2(13.33 \%)$ & \\
\hline Moderate & - & $1(6.67 \%)$ & \\
\hline Severe & - & $12(80 \%)$ & \\
\hline \multicolumn{4}{|l|}{ Displacement, n (\%) } \\
\hline $24 \mathrm{~h}$ after operation & $1(6.67 \%)$ & $11(73.33 \%)$ & $<0.001^{\star}$ \\
\hline $48 \mathrm{~h}$ after operation & $1(6.67 \%)$ & $14(93.33 \%)$ & $<0.001^{\star}$ \\
\hline \multicolumn{4}{|l|}{ Change dressing, $\mathrm{n}(\%)$} \\
\hline $24 \mathrm{~h}$ after operation & 0 & $1(0-3)$ & 0.001 \\
\hline $48 \mathrm{~h}$ after operation & 0 & $1(0-4)$ & $<0.001$ \\
\hline \multicolumn{4}{|l|}{ NRS } \\
\hline $24 \mathrm{~h}$ after operation & $2(0-7)$ & $2(0-8)$ & 0.539 \\
\hline $48 \mathrm{~h}$ after operation & $2(0-7)$ & $2(0-7)$ & 0.806 \\
\hline \multicolumn{4}{|c|}{ Post-operative morphine consumption (mg) } \\
\hline \multicolumn{4}{|l|}{ Median (IQR) } \\
\hline $24 \mathrm{~h}$ morphine consumption & $3(0-5)$ & $2(0-6)$ & 0.512 \\
\hline $48 \mathrm{~h}$ morphine consumption & $0(0-5)$ & $0(0-3)$ & 0.713 \\
\hline Satisfaction score, mean $\pm S D$ & $9.13 \pm 1.13$ & $9.07 \pm 1.28$ & 0.881 \\
\hline
\end{tabular}

H: Hour, IQR: Interquartile range, NRS: Numeric rating scale, SD: Standard deviation 


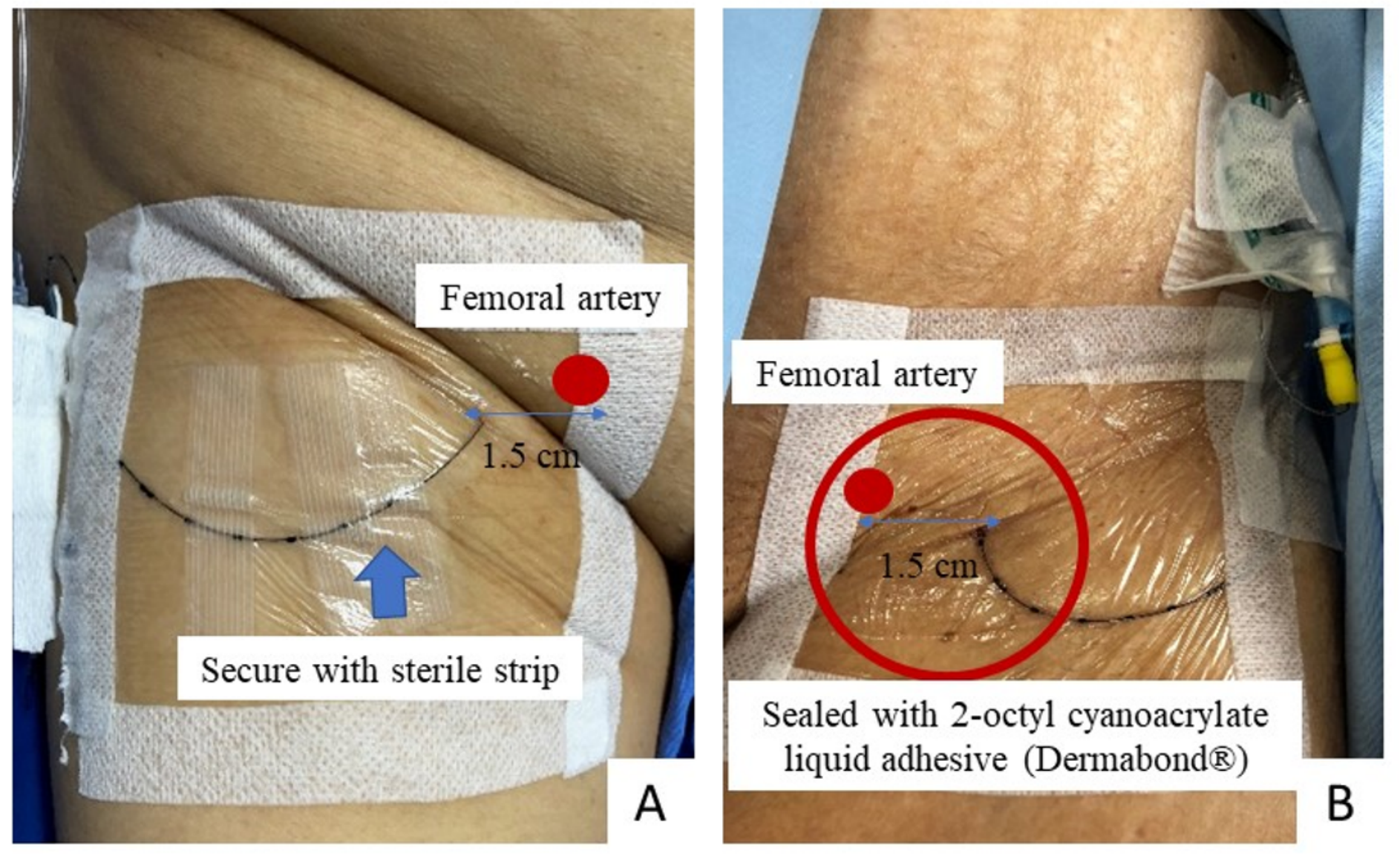

Figure 1

(A) Catheter is secured with sterile strip at right groin. (B) Catheter is secured with 2-octyl cyanoacrylate liquid adhesive (Dermabond $\AA$ ) at left groin. 


\section{Enrollment $(\mathrm{n}=34)$}

\section{Assessed for eligibility $(n=34)$}

Randomized $(\mathrm{n}=30)$

Exclude $(\mathrm{n}=4)$

- Not meeting inclusion criteria $(\mathrm{n}=2)$

- Infection at groin area $(\mathrm{n}=1)$

- History allergy to local anesthesia $(\mathrm{n}=1)$

- Declined to participate $(\mathrm{n}=2)$

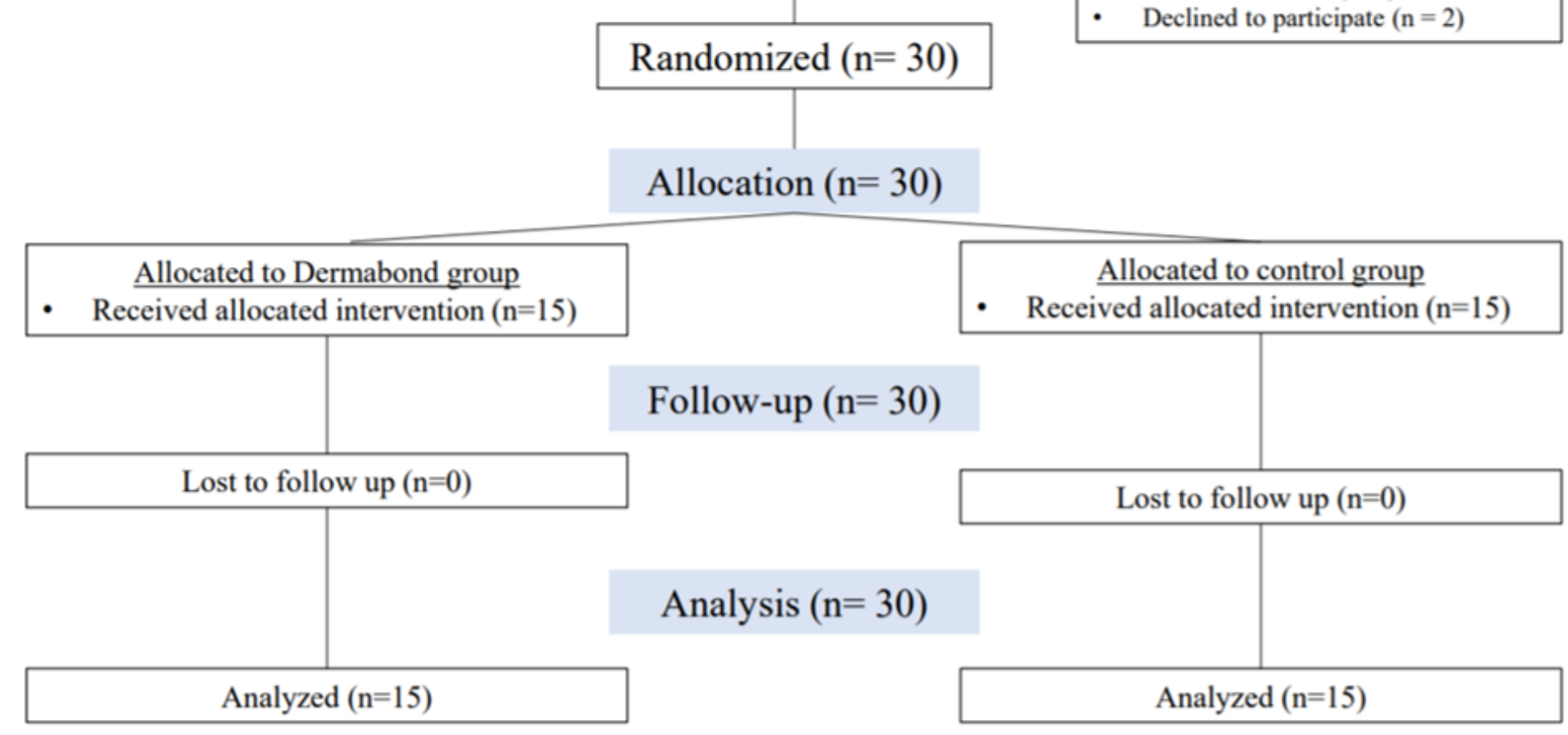

Figure 2

CONSORT diagram showing the flow of the study participants

\section{Supplementary Files}

This is a list of supplementary files associated with this preprint. Click to download.

- CONSORT2010Checklist3.doc 